\title{
Marco de referencia para la integración de la contabilidad social en la gestión estratégica de las empresas de Economía Social
}

\section{Alfonso Echanove Franco}

RESUMEN: La presión social e institucional al tejido empresarial por generar y evidenciar un valor más allá de sus resultados económicos se ha incrementado en los últimos años. A las demandas de información sobre los impactos positivos y negativos de la actividad empresarial se suma ahora la necesidad de plantear e implantar acciones de impacto social positivo. En este contexto las empresas de Economía Social se enfrentan a un reto importante. Por su naturaleza cabría atribuirles una mayor generación de valor social y, sin embargo, esta no puede evidenciarse exclusivamente a través de la formulación de sus aspiraciones sociales. Surge la necesidad de integrar de forma diferenciada la generación y distribución de valor social en su gestión estratégica. Lo anterior se sustenta en el presente artículo en un análisis comparativo entre empresas de Economía Social y empresas mercantiles sobre la gestión del valor social en dos hitos de sus procesos de gestión estratégica: la declaración de la intención estratégica y la medición de resultados. Del análisis se infiere la necesidad de contar con sistemas de información social robustos, que permitan la trazabilidad entre la definición del propósito de la organización y sus resultados y por ende, la comparativa entre organizaciones. La aplicación del modelo poliédrico de contabilidad social se ha considerado adecuada en estudios previos para la medición y comunicación del valor social generado por organizaciones de la Economía Social. Con el presente artículo se pretende incorporar un elemento novedoso al proponer su integración en los procesos de gestión estratégica.

PALABRAS CLAVE: Valor social, estrategia, gestión estratégica, contabilidad social, modelo poliédrico.

CLAVES ECONLIT: L20, M14, M40, P41.

Cómo citar este artículo/How to cite this article: ECHANOVE, A. (2020): "Marco de referencia para la integración de la contabilidad social en la gestión estratégica de las empresas de Economía Social", CIRIEC-España, Revista de Economía Pública, Social y Cooperativa, 100, 207-237. D0I: 10.7203/ CIRIEC-E.100.18118.

Correspondencia: Alfonso Echanove Franco, aetxanobe@lksnext.com, Universidad del País Vasco/ Euskal Herriko Unibertsitatea, ORCID: 0000-0001-9498-9670 


\section{Expanded abstract}

\section{Reference framework for the integration of social accounting in the strategic management of social economy enterprises}

\section{Context and objective}

The social and institutional pressure on businesses to generate and demonstrate a value that goes beyond their economic results has increased in recent years. In addition to the demands for information on the impacts of the business activity, there is now a need to propose and implement actions with a positive social impact. In this context, Social Economy enterprises face an important challenge. By their nature, a greater generation of social value could be expected, and yet this cannot be evidenced exclusively through the formulation of their social aspirations. Furthermore, commercial companies have internalized the need to manage and communicate the social value they generate, adopting concepts and lexicon of a social nature in their presentations and activity reports. In any case, there may be an important gap between what has been declared and what has been achieved. It is argued that resources are not really assigned to generate social value or that social value is only treated by some companies as a marketing element.

This points to the need to improve the social value management from a strategic point of view, integrating it into the enterprises' strategic management process. This process is crucial in any organisation and should not be limited to the design phase but also contemplate the implementation of and monitoring of the results. As long as this integration is effective, Social Economy enterprises will be able to demonstrate the coherence between the definition of their purpose, the allocation of resources and their actual performance. In this context, the objective of this article is to provide a reference framework for the integration of social accounting in the strategic management process of Social Economy enterprises. This will, in turn, enable companies to incorporate social value as an element of their strategy in an effective manner.

\section{Design and methodology}

The analysis of the theoretical framework starts from the evolution of the concepts of Strategy, strategic process and strategic management process. From that point on, the analysis is structured around two key moments of the strategic management cycle: formulation of the strategic intent, allocation of resources and results measurement. All three constitute a reference model in which the need for coherence between the strategic intention and the results obtained is highlighted.

On the basis of this framework, the analysis and discussion are developed. A comparative analysis has been undertaken between groups of Social Economy and commercial enterpris- 
es from five industrial sectors according to the National Classification of Economic Activities (CNAE) codes, and from two perspectives. On the one hand, a semantic-pragmatic analysis of the strategic intention, as stated in their websites, of a sample of 60 companies belonging to both groups. The information has been analysed following three main dimensions: production and commercial activity, social commitment and enterprise model.

On the other hand, a quantitative study based on data from financial profiles has been implemented. This has allowed the comparative analysis of the distribution of value added of a sample of Social Economy and commercial enterprises, following the Added Value Statement model proposed by the Spanish Association of Accounting and Business Administration (AECA). This analysis seeks to identify evidence of the coherence between some differentiating factors of the Social Economy and the actual results obtained.

\section{Results and implications}

As far as the analysis of the strategic intention of the sample is concerned, the results show that both Social Economy and commercial enterprises tend to incorporate equivalent messages in their strategic statements as regards their production and commercial activity and their commitment with Society. The companies in both groups, mainly in the manufacturing and agri-food sectors, introduce competitiveness, profitability, quality, innovation, technological capacity and efficiency in their declarations. Besides, concepts such as sustainability, responsibility with regard to social and environmental issues or concern for employment are also found indistinctly in commercial and Social Economy enterprises. In short, those companies that carry out a market activity tend to incorporate economic and social criteria in a homogeneous way as a part of the strategic intention that they state in their corporate presentations. Although the declaration of a social purpose is manifested as necessary and is widely used in large companies, it is not a proof of the actual generation of social value nor does it guarantee its fulfilment. The biggest differences between the strategic intent of Social Economy and commercial enterprises are found when facing the third dimension of the analysis -enterprise model and employment. Thus, cooperatives mention their cooperative nature as a distinctive factor and specifically adhere to Social Economy principles.

The analysis of the financial data allows to conclude that the structure of distribution of added value in Social Economy enterprises is different insomuch as the remuneration to the labour factor is proportionally higher than the remuneration to any of the other production factors if compared to the structure of distribution of added value in commercial companies. This might be a proof of the distribution of the benefits of the activity on the basis of the contribution of work, and not capital, which is one of the Social Economy principles. However, it would be difficult to report on other principles only taking into account the financial, economic or market information which is usually included in the strategic analyses. There is no comparable information on the social value generated, which makes it difficult to manage and guarantee the alignment between the strategic intention and the results obtained. The application of the polyhedral model of Social Accounting arises as a potential tool to be integrated in this reference framework as it may help to bridge the gaps between strategic intent and strategic performance. 
It is concluded that in a global framework in which the United Nations Agenda is present in institutional and business environments and the correlation between business activity and sustainability has become more evident, Social Economy companies need to integrate social value in their strategic management in a more professional and efficient manner. In the future, a research path is opened for the development of a model that, based on the reference framework proposed here, incorporates the key issues, processes and tools for the effective integration of social value in the strategic management of organizations that aspire to generate a social value, in such a way that it can be measured, communicated and managed. Thus, institutions and the society as a whole will be able to evaluate and recognize the contribution of Social Economy to the generation of social value.

\section{Contribution and originality}

Among the issues to be included in the proposed framework is the need for a social information system that can be integrated into the companies' information systems and that allows comparability between organizations. In this sense, recent studies have analysed the adequacy of social accounting to reflect the social value generated by Social Economy enterprises.

This article provides an innovative and practical vision by adding new perspectives of analysis and providing the framework for integrating social value into a company's strategic management process by means of the application of the polyhedral model of social accounting.

\section{Limitations}

In the first place, the analysis of the structure of generation and distribution of added value has been limited to a single year, therefore lacking an analysis of the evolutionary dynamics of the benchmark indicators. On the other hand, the CNAE sectors analysed can include entities which are not easily comparable as the number of Social Economy enterprises in some of the sectors is low and its dimension is by far smaller than that of commercial companies in the same sectors. Also, the analysis of the Social Economy enterprises has been limited to cooperatives and labour societies as the most representative Social Economy enterprises in Spain; leaving out, therefore, other business initiatives such as Special Employment Centers and Insertion Companies, unless they are constituted as cooperatives or labour societies. Finally, the analysis has been limited to five sectors of activity, among them the most representative ones for Social Economy in Spain. Nonetheless, the extension to other sectors might also yield some different results and perspectives of analysis.

Keywords: Social value, strategy, strategic management, social accounting, polyhedral model 


\section{Introducción}

La distinción más básica entre las empresas sociales y las empresas mercantiles considera el fin último que persiguen. Así, la empresa social persigue un fin social y la empresa mercantil persigue un fin económico. De acuerdo con este criterio, no obstante, son pocas las empresas que persiguen exclusivamente uno u otro fin y la distinción más bien permite la conceptualización de las organizaciones en una escala de múltiples niveles cuyos extremos estarían representados por empresas puramente mercantiles o entidades puramente sociales (Austin et al., 2012).

La generación de valor social, en todo caso, no es una preocupación exclusiva de las empresas sociales. La presión institucional a todo tipo de empresas por generar valor más allá de los resultados financieros ha crecido como resultado de profundos cambios sociales. Además, existe una demanda cruzada entre las organizaciones y la sociedad por disponer de una mejor información sobre el impacto social de sus actividades (Retolaza \& San-Jose, 2018). La transposición en los diferentes Estados miembro de la Unión Europea de la Directiva 2014/95 sobre la divulgación de información no financiera e información sobre diversidad por parte de determinadas grandes empresas y determinados grupos supone la concreción legislativa de estas tendencias sociales. Se espera que las empresas sean más transparentes y que esto ayude a que sean a su vez más resilientes y tengan mejores rendimientos financieros y no financieros, correlacionando así la información veraz y transparente con la sostenibilidad. Para mantener los mecanismos necesarios para impulsar la sostenibilidad, los diferentes agentes que actúan en el mercado necesitan contar con información útil y creíble (Etxebarria, 2009).

Las empresas de Economía Social alinean objetivos económicos, sociales y medioambientales al representar un modelo de empresa transparente y participativo, que genera empleo de calidad y contribuye a un crecimiento económico inclusivo, respetuoso con el medio ambiente. "En un momento en el que la sociedad está aspirando a construir un modelo de organización y de desarrollo económico más responsable, inclusivo y sostenible, la Economía Social responde a la demanda creciente de empresas más responsables, transparentes, donde la ciudanía participe y en las que se promueva la igualdad de oportunidades" (Pedreño, 2017).

Siendo, por lo tanto, un modelo que aporta un valor social diferencial; sin embargo, no resulta fácil evidenciarlo ni gestionarlo. La medición del valor social es compleja por las diferentes percepciones de lo que es en sí, y lo es así mismo la gestión del valor social desde una perspectiva estratégica ya que la Estrategia está relacionada con la fijación y seguimiento de objetivos (Chandler, 1962; Andrew, 1971; King et al., 1978) y los objetivos deben poder ser medidos (Norton \& Kaplan, 1996). Se ha atribuido al valor social la no-cuantificabilidad (Austin et al., 2012), lo que hace difícil para las empresas con fines sociales medir, gestionar e integrar en su gestión estratégica el valor social que generan.

En los últimos años se han desarrollado diferentes investigaciones en las que se trata de relacionar información financiera con los valores de la Economía Social. Así, Iturrioz et al. (2019) vinculan Economía Social y RSC y concluyen que el tamaño y la liquidez presentan una mayor correlación significativa con respecto a la información publicada en los informes de RSC mien- 
tras que la transparencia no afecta a la rentabilidad económica ni a la financiera. Lazcano \& San-Jose (2019) proponen desde la praxis de un grupo empresarial de Economía Social que la contabilidad social podría aportar información del valor social generado que no proporciona la información financiera.

En este contexto, el objetivo de este artículo es proponer un marco de referencia para la integración del valor social en la gestión estratégica de las empresas de Economía Social. Se contemplan diferentes perspectivas de análisis y se propone el modelo poliédrico de contabilidad social como instrumento para cubrir la gestión del purpose gap (Gast et al., 2020) entre la intención estratégica de las organizaciones y los resultados obtenidos. La utilidad del modelo poliédrico se ha evaluado en diferentes investigaciones desde 2012 y este artículo aporta una visión novedosa al contemplar su lógica de integración en el ciclo completo de la gestión estratégica, que abarca desde la formulación de la intención estratégica al seguimiento de los resultados.

La Estrategia es el elemento que ayuda a centrar la atención en las finalidades de la organización (Miller et al., 1996) y la coherencia en la gestión del ciclo estratégico evita el riesgo de pérdida de foco en lo que para una organización puede ser esencial. En la medida en que las empresas integren el valor social en su gestión estratégica, entendida como el proceso que va desde la formulación a la implantación de la estrategia (Francés, 2006) será más posible pasar de iniciativas puntuales de información a una acción empresarial coherente con su propósito. Drucker (1986: p.18) apuntaba a que "la gestión es responsable de producir los resultados para los que una empresa se ha creado". En el ámbito de la gestión empresarial como disciplina de estudio ha prevalecido durante décadas una orientación a la generación de valor financiero para los accionistas. Friedman (1970), manifestaba que las empresas existen para generar valor para sus accionistas, lo que ha sido un paradigma ampliamente interpretado, contrastado y debatido. Con todo, la integración del valor social en la gestión estratégica ha tenido un desarrollo considerablemente menor al de la integración del valor financiero. Conceptos como la Responsabilidad Social Corporativa (RSC) o el valor compartido (Porter et al., 2006) han emergido de forma discontinua como elementos de reflexión y debate.

El artículo parte de una exposición del marco teórico necesario a fin de establecer el alcance con el que hacemos referencia al proceso de gestión estratégica en una organización. A partir de ahí, el análisis y discusión giran en torno a dos de los principales hitos de dicho proceso, que marcan el inicio y el fin del mismo: la formulación de la intención estratégica y la obtención de resultados. Considerando el carácter cíclico del proceso, surge así un marco de referencia y diferentes cuestiones; entre ellas, la contabilidad social como respuesta a la necesidad de disponer de información social para la toma de decisiones estratégicas coherentes con la intención de generar valor social.

Por otro lado, la necesidad de dicha información se fundamenta así mismo a lo largo del artículo en un análisis comparativo entre una muestra de empresas de Economía Social (cooperativas y sociedades laborales) y otra de empresas mercantiles, aportando una doble perspectiva: el análisis de la declaración de su intención estratégica y el de los resultados obtenidos. Para la primera perspectiva se ha utilizado un análisis semántico pragmático a partir de la 
información estratégica de las websites de 60 empresas de Economía Social y mercantil entre las que están representadas las de mayor cifra de negocios de 2018 en 5 sectores de actividad CNAE. La segunda perspectiva, con la que se analiza si la información financiera permite identificar el valor social diferencial de las empresas de Economía Social de los grupos analizados y, por ende, gestionarlo estratégicamente; se basa en datos secundarios referidos a las cuentas anuales de 2018, obtenidos de la base de datos SABI.

\section{Marco teórico}

Desde que la estrategia empresarial experimentase un auge importante como objeto de estudio a mediados del siglo XX, las definiciones de estrategia han sido muy diversas. Una de las más influyentes es la de Chandler (1962), que describía la estrategia como "la determinación de las metas y objetivos de una empresa a largo plazo, implica el diseño de los cursos de acción y la asignación de los recursos necesarios para alcanzar dichos objetivos y metas". Surge desde aquí la visión de la estrategia como un proceso, en el que se pone el énfasis en la planificación, la toma de decisiones y la asignación de recursos con una mirada a largo plazo.

Mintzberg (1990) aborda un análisis pormenorizado de la forma en que este proceso se lleva a cabo en las que identifica como las escuelas de pensamiento estratégico surgidas en las décadas anteriores. El autor profundiza especialmente en las de diseño, planificación y posicionamiento, que considera escuelas prescriptivas, y en su análisis añade a lo que llama el proceso estratégico elementos de contexto, organización y cultura.

A finales del siglo XX se añade un elemento más en el intento por alinear la operativa de las empresas con su estrategia, al incorporar los mecanismos de control en la implantación de la estrategia. Esto nos lleva al concepto del proceso de gestión estratégica que guía nuestro artículo. El proceso se asocia al llamado ciclo estratégico: Definición, planificación -acciones, responsables y recursos- y seguimiento y control (Francés, 2006) o formulación, implantación y control (Pearce \& Robinbson, 2011). En este mismo sentido se habla de los elementos nucleares del proceso de gestión estratégica: la definición de la posición estratégica, la toma de decisiones y la implantación de la estrategia -Strategy into action (Johnson et al., 2012). En suma, el proceso de gestión estratégica persigue la coherencia entre la declaración de una intención, la asignación de recursos y los resultados que obtiene una organización.

Austin et al. (2012) identifican tres modos naturales de diferenciar la empresa mercantil y la social: Su finalidad principal declarada en su misión, los criterios y mecanismos de asignación de recursos y la forma de medir los resultados. Lo anterior nos lleva a identificar un continuum en la potencial gestión de valor económico o social, que resulta armónico con los momentos clave del ciclo estratégico. Entendiendo la correspondencia entre los aspectos de diferenciación planteados, las fases del proceso de gestión estratégica y los elementos que en cada una de ellas se desarrollan, obtenemos un marco de referencia para estructurar la discusión y el análisis (ver Figura 1), en el que se destaca la necesidad de coherencia entre la intención estratégica y los resultados obtenidos. 


\section{Figura 1. Elementos para orientar la gestión del valor y su correspon- dencia con el proceso de gestión estratégica}

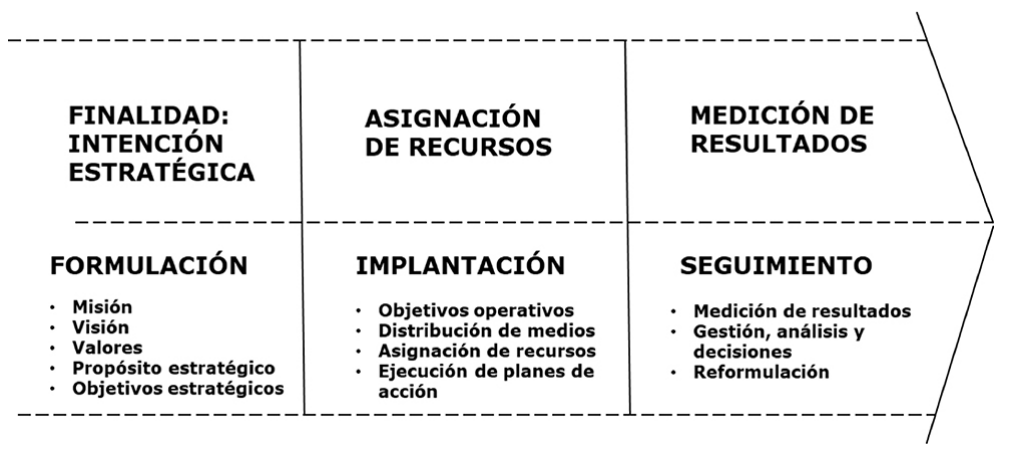

Fuente: Elaboración propia.

\section{La declaración de la intención estratégica}

El punto de partida del ciclo de gestión estratégica es la declaración de la intención estratégica (strategic intent), que fija la ambición, el objetivo que merece un esfuerzo y un compromiso (Hamel \& Prahalad, 1989). Es un elemento estable en el tiempo y motivador al comunicar el valor que genera la empresa. Se ha identificado con otros conceptos como la orientación estratégica (Hitt et al., 1995), la declaración de la cultura corporativa (Gast et al., 2020), el propósito (Hamel \& Prahalad, 2005), la Misión, la Visión y los Valores (Gratton, 1994; Odita et al., 2015) o los compromisos y objetivos de primer nivel (Brand, 2012).

Desde esta primera perspectiva se ha argumentado que las diferencias entre las empresas sociales y mercantiles se manifiestan en que mientras que estas se preocupan principalmente por obtener beneficios privados, aquellas están más preocupadas por generar valor social (Trevis Certo et al., 2008). Los mismos autores reconocen que en la ejecución de sus procesos las empresas mercantiles pueden generar valor social y las empresas sociales pueden generar beneficios privados pero concluyen que a pesar de estos beneficios secundarios, las organizaciones mercantiles y sociales están impulsadas por misiones muy diferentes.

En la práctica, sin embargo, las misiones y otras declaraciones para expresar la intención estratégica de organizaciones de diferentes tipos han tendido a evolucionar hacia declaraciones de un carácter más híbrido. Por un lado, muchas empresas han hecho mucho por mejorar el impacto social y ambiental de sus actividades, basándose en que la empresa y la sociedad son claramente interdependientes y el propósito de las empresas debe ser la creación de valor compartido (Porter \& Kramer, 2006). Por otro lado, frente a las posiciones que subrayan el fin exclusivamente social de la empresa social, se ha planteado que la empresa social es híbrida 
por naturaleza, como lo indica su propia denominación a través de la unión de los conceptos de empresa y social (Grassl, 2012).

La tendencia se ha intensificado en los últimos años tras la crisis financiera de 2008 y se han retomado o incluso fortalecido los conceptos vinculados a la Responsabilidad Social Corporativa (RSC), tanto en el ámbito empresarial como en el debate institucional, incorporándolos como fines propios de las empresas. De acuerdo con las investigaciones de Lauesen (2013), aunque el concepto de Responsabilidad Social Corporativa puede encontrar sus raíces tras la II Guerra Mundial, es a finales del siglo XX cuando cobra una especial importancia como objeto de estudio y la producción científica crece de forma exponencial. Por un lado, se han destacado los vínculos entre la Economía Social y la RSC, debido a que la Economía Social, y en especial, las cooperativas, representan un tipo de empresa basado en los mismos valores que la RSC (de Castro, 2006; Iturrioz et al., 2019). Por otro lado, la RSC ha ganado impulso durante los últimos años tras la adopción en 2015 de los Objetivos de Desarrollo Sostenible (ODS) de Naciones Unidas, enmarcados en la Agenda global 2030, que cuenta con un importante respaldo institucional de los Estados firmantes y que parece haber movilizado el entorno empresarial. Si bien la iniciativa todavía no tiene un recorrido suficiente como para poder evaluar su impacto real en la estrategia y los modelos de gestión de las empresas, sí se percibe un impacto al menos formal en el crecimiento de la referencia a los ODS en informes de gestión, memorias de sostenibilidad y otros instrumentos de gestión y comunicación. Así mismo, el número de empresas adheridas a las Redes nacionales del Pacto Mundial sigue creciendo año tras año ${ }^{1}$.

\section{Asignación de recursos}

La base conceptual de esta fase del proceso está en la teoría de recursos y capacidades (Peteraf, 1993; Barney, 1997) que defiende que la heterogeneidad de recursos y capacidades existentes en las empresas determina las diferencias de resultados entre unas y otras.

Asumiendo una perspectiva fundamentalmente económica, la asignación de recursos se asoció durante un tiempo a la definición de los presupuestos, fundamentados en métodos cuantitativos de valoración de las inversiones. Se trataba, por lo tanto, de un ejercicio de planificación financiera, cuyo estilo y alcance fue puesto en cuestión en entornos de mayor presión hacia los equipos directivos (Hope et al., 2003) que demandaban formas de liderazgo y gestión más ágiles y adaptativas. En esta línea, el desarrollo de otros paradigmas demanda contemplar otro tipo de recursos además de los económicos y otras dimensiones que aporten flexibilidad en el desarrollo de las actividades empresariales y ajuste continuo al perfil y misión de la empresa y el contexto estratégico en el que opera. De aquí se deriva la concepción de la estrategia como un proceso iterativo de asignación de recursos (Bower et al., 2005), en el que las

1. La Red Española del Pacto Mundial vio incrementado el número de entidades asociadas entre 2010 y 2018 de 241 a 661 y en los últimos dos años la cifra se ha elevado hasta 1.508 (Fuente: Red Española del Pacto Mundial de Naciones Unidas, 2020). 
actividades de cada nivel de dirección contribuyen al aprendizaje colectivo de la organización y conforman su estrategia.

En línea con lo anterior, Maritan \& Lee (2017) reconocen que "soprendentemente no hay un gran cuerpo de literatura específica sobre la asignación de recursos financieros, físicos, tecnológicos y humanos para soportar las estrategias de negocio". Incorporando un aspecto adicional, la asignación de recursos se ha analizado con respecto al liderazgo de la dirección (Chin et al., 2013; Gupta et al., 2018), encontrando una relación entre la ideología de la persona que ocupa el puesto de CEO y la asignación de recursos a través de las diferentes unidades organizativas. Y profundizando en la relación con la flexibilidad estratégica Song et al. (2020) identifican la asignación de recursos tecnológicos y capacidades para la gestión de alianzas como elementos clave para mantener la flexibilidad estratégica en contextos de incertidumbre.

La coherencia estratégica demandaría que los recursos utilizados por las empresas que declaran un fin social estuvieran vinculados a tal fin social. En el marco de una orientación al valor social, se asume que las motivaciones o finalidades de las personas varían al evaluar la asignación de recursos (Murphy et al., 2011) y elegirían el beneficio social frente al propio. De acuerdo con la teoría de recursos y capacidades, su disposición podría generar una ventaja competitiva en la medida en que se desplegasen en el marco de una estrategia bien concebida (Collis et al., 1995).

Sin embargo, se ha argüido que en el caso de las empresas sociales, las personas que las gestionan pueden estar tan focalizadas en encontrar recursos económicos para posibilitar el crecimiento de la organización que la creación de valor social queda desdibujada. Los recursos económicos son un medio para desarrollar una propuesta de valor social pero se requiere afrontar su captación con una visión más amplia (Austin et al., 2012).

\section{Medición de resultados}

De acuerdo con el esquema sugerido, la tercera perspectiva que potencialmente distingue la actividad empresarial con un fin social de la puramente mercantil es la medición de los resultados obtenidos. En el proceso de gestión estratégica, la medición de resultados vincula los planos estratégico y operativo, contribuyendo a una efectiva puesta en acción de la estrategia. Los sistemas de medición de resultados estratégicos de una organización posibilitan la integración entre la estrategia y las operaciones (Chenhall, 2005).

Siguiendo con la argumentación anterior, si la estrategia de una organización está orientada a la generación de valor social, poner en marcha esta estrategia de forma efectiva requerirá un sistema de medición de tal valor. Sin embargo, Austin et al. (2012) ya alertan de que el reto de medir el cambio social generado a través de las actividades empresariales con un fin social es grande debido a la no-cuantificabilidad, la multicausalidad, las dimensiones temporales y las diferencias de percepción sobre el impacto social creado.

Las empresas que desarrollan una actividad de mercado tienden a medir y hacer seguimiento de una serie de indicadores estandarizados como las ventas, el valor añadido, la rentabilidad económica o la rentabilidad financiera, entre los más habituales. En la medida en que las 
métricas de resultados económico-financieros están estandarizadas, pueden ser reconocidas y valoradas por empresarios e inversores (Trevis Certo et al., 2008). Estos indicadores no sólo proporcionan la información necesaria para valorar el cumplimiento de los objetivos de la empresa sino que el análisis de su evolución, en coherencia con las aspiraciones de la organización, es el primer input para formular estrategias de crecimiento, eficiencia o mercado. Incorporando otro tipo de indicadores acordes con las claves del modelo de negocio de una empresa, se dispone de la información necesaria para la gestión estratégica de la organización desde las perspectivas corporativa, competitiva o funcional. En suma, el uso de indicadores permite a una organización determinar si está alcanzando sus objetivos (Marsden et al., 2006)

En esta línea, la creación y publicación del cuadro de mando integral (Norton et al., 1996) supuso un avance importante para cubrir el gap existente entre la estrategia y la operativa. Con el cuadro de mando integral se aportó un enfoque sistémico para el seguimiento de los resultados de la empresa, incorporando la necesidad de fijar objetivos relativos a cuatro perspectivas (junto a la financiera, las de clientes, procesos internos y aprendizaje). Otra de las aportaciones del cuadro de mando integral es su perspectiva temporal. Mientras que los indicadores más tradicionales tienden a centrarse en el análisis del pasado el cuadro de mando integral se convierte además en el eje de la estrategia actual y futura de la organización. Facilita la gestión estratégica en la organización al contemplar, por lo tanto, su visión sistémica y el análisis de la perspectiva temporal, dos de los ejes para promover el pensamiento estratégico en las organizaciones (Lietdka, 1998).

En relación con cada una de las cuatro perspectivas del cuadro de mando integral, su aplicación práctica en numerosas organizaciones ha permitido desarrollar métricas estandarizadas que permiten a la empresa conocer y evaluar de forma comparativa ratios de dependencia de clientes o proveedores e indicadores de innovación o productividad de diferentes procesos, por nombrar algunos. A los indicadores que provienen de la contabilidad financiera se añaden otros indicadores estratégicos o de gestión que constituyen inputs fundamentales para el desarrollo de estrategias funcionales. Incluso en algunos casos se sugiere una correlación entre algunas medidas de los sistemas de medición y la rentabilidad de una organización (Azofra et al., 2003)

Sin embargo, las mediciones de generación de valor social están menos estandarizadas y cada organización las adapta a su realidad, resultando complicada su valoración, su comparación con entidades similares y, en suma, la gestión estratégica de tal valor social.

\section{Metodología y muestra}

El marco de referencia está estructurado en torno a las tres grandes perspectivas del proceso de gestión estratégica -la declaración de la intención estratégica, la asignación de recursos y el seguimiento de los resultados- y centraremos la discusión en torno al inicio y al fin del proceso y la coherencia entre estos dos momentos: la formulación de la intención estratégica y el seguimiento de los resultados. 
Para ello, se ha realizado un análisis comparativo entre grupos de empresas de Economía Social y empresas mercantiles de cinco sectores industriales según códigos CNAE y desde dos perspectivas. En primer lugar, el análisis comparado de la intención estratégica de una selección de empresas pertenecientes a ambos grupos a partir de un enfoque semántico-pragmático (Molero, 2003), explicando así los significados del léxico utilizado y los conceptos inherentes en la comunicación de las empresas de su intención estratégica. Se ha recurrido a las presentaciones de las websites corporativas, toda vez que las websites se han confirmado como un soporte clave de la comunicación estratégica (Pintado, 2017). 


\title{
Tabla 1. Ficha de análisis. Análisis comparativo de la intención estra- tégica de un grupo de empresas de Economía Social y un grupo de empresas mercantiles
}

\section{Identificar los elementos clave de la intención estratégica de empresas de Economía Social y empresas mercantiles en cinco sectores, de acuerdo con la clasificación CNAE2009}

\author{
Empresas Empresas activas en España en diferentes sectores de actividad, analizadas en \\ objeto de dos grupos: \\ análisis
}




\begin{tabular}{|c|c|c|c|}
\hline Grupo CNAE & Descripción corta & Tipología & Muestra \\
\hline \multirow{2}{*}{ GRUPO A } & \multirow{2}{*}{ Agroalimentarias } & ECO SOCIAL ${ }^{1}$ & 8 \\
\hline & & $S A+S L^{2}$ & 8 \\
\hline \multirow{2}{*}{ GRUPO B } & \multirow{2}{*}{ Extractivas } & ECO SOCIAL ${ }^{1}$ & 0 \\
\hline & & $S A+S L^{2}$ & 2 \\
\hline \multirow{2}{*}{ GRUPO C } & \multirow{2}{*}{ Manufactureras } & ECO SOCIAL ${ }^{1}$ & 9 \\
\hline & & $S A+S L^{2}$ & 9 \\
\hline \multirow{2}{*}{ GRUPO D } & \multirow{2}{*}{ Energía y gas } & ECO SOCIAL ${ }^{1}$ & 6 \\
\hline & & $S A+S L^{2}$ & 6 \\
\hline \multirow{2}{*}{ GRUPO E } & \multirow{2}{*}{ Agua, saneamiento y gestión de residuos } & ECO SOCIAL ${ }^{1}$ & 6 \\
\hline & & $S A+S L^{2}$ & 6 \\
\hline \multirow{2}{*}{ TOTAL } & & ECO SOCIAL ${ }^{1}$ & 30 \\
\hline & & $5 A+5 L^{2}$ & 30 \\
\hline
\end{tabular}

1. Economía Social (Cooperativas y Sociedades Laborales)

2. Sociedades Anónimas y Sociedades Limitadas

Fuente: Elaboración propia a partir de base de datos SABI y Clasificación Nacional de Actividades Económicas (CNAE - 2009).

En un momento posterior del análisis, se ha abordado un estudio cuantitativo basado en datos de perfiles financieros y el análisis de distribución del valor agregado entre los factores de producción (Ver Tabla 3) de un conjunto más amplio de empresas de Economía Social y empresas mercantiles (Ver Tabla 4) correspondientes a los cinco sectores analizados. 


\section{Tabla 3. Análisis comparativo de la distribución del valor agregado en- tre empresas de Economía Social y empresas mercantiles}

\section{Objetivo \\ Identificar diferencias en la distribución del valor agregado y, específicamente, en la retribución del factor trabajo entre empresas de Economía Social y empresas mercantiles.}

\begin{tabular}{|c|c|}
\hline $\begin{array}{l}\text { Empresas objeto de } \\
\text { análisis }\end{array}$ & $\begin{array}{l}\text { Empresas activas con actividad productiva en España en } 2018 \text { en } \\
\text { cinco sectores de actividad, analizadas en dos grandes grupos } \\
\text { configurados de acuerdo con su forma jurídica: } \\
\text { Cooperativas y Sociedades Laborales (empresas de Economía Social) } \\
\text { Sociedades anónimas y Sociedades Limitadas (empresas } \\
\text { mercantiles) }\end{array}$ \\
\hline Metodología & $\begin{array}{l}\text { Análisis comparativo de la distribución del valor agregado entre } \\
\text { dos grupos de empresas (Economía Social y mercantiles) de cinco } \\
\text { sectores de actividad. En cada uno de los sectores se compara la } \\
\text { distribución del valor agregado entre los diferentes factores de } \\
\text { producción y la media aritmética de las variables obtenidas. }\end{array}$ \\
\hline Fuentes de información & $\begin{array}{l}\text { Información obtenida de las cuentas de explotación depositadas por } \\
\text { las empresas objeto de estudio en el año } 2018 \text { y disponibles en la } \\
\text { base de datos SABI }\end{array}$ \\
\hline $\begin{array}{l}\text { Indicadores del perfil } \\
\text { financiero analizados }\end{array}$ & $\begin{array}{l}\text { Empleo, Valor agregado, Gastos de personal, Gastos financieros } \\
\text { y asimilados, Impuestos sobre Sociedades, Dotaciones para } \\
\text { amortización de inmovilizado y Resultado del ejercicio. }\end{array}$ \\
\hline Tamaño de la muestra & $\begin{array}{l}\text { Se ha considerado el grupo entero de empresas de las características } \\
\text { anteriores cuya información está disponible en la base de datos SABI. } \\
\text { (Ver tabla } 4 \text { para la cuantificación de la muestra) }\end{array}$ \\
\hline $\begin{array}{l}\text { Fecha de disponibilidad } \\
\text { de la información }\end{array}$ & $\begin{array}{l}\text { Análisis realizado sobre la información disponible entre el } 20 \text { de } \\
\text { enero y el } 15 \text { de febrero de } 2020 \text {, referida al ejercicio } 2018\end{array}$ \\
\hline
\end{tabular}

Fuente: Elaboración propia. 
Tabla 4. Muestra por sector de actividad (Grupo CNAE)

\begin{tabular}{|c|c|c|c|}
\hline Grupo CNAE & Descripción & Tipología & Muestra \\
\hline \multirow{2}{*}{ GRUPO A } & \multirow{2}{*}{ Agroalimentarias } & ECO SOCIAL ${ }^{1}$ & 418 \\
\hline & & $S A+S L^{2}$ & 16.786 \\
\hline \multirow{2}{*}{ GRUPO B } & \multirow{2}{*}{ Extractivas } & ECO SOCIAL & 6 \\
\hline & & $S A+S L^{2}$ & 1.135 \\
\hline \multirow{2}{*}{ GRUPO C } & \multirow{2}{*}{ Manufactureras } & ECO SOCIAL ${ }^{1}$ & 1.387 \\
\hline & & $S A+S L^{2}$ & 56.838 \\
\hline \multirow{2}{*}{ GRUPO D } & \multirow{2}{*}{ Energía } & ECO SOCIAL ${ }^{1}$ & 30 \\
\hline & & $S A+S L^{2}$ & 6.443 \\
\hline \multirow{2}{*}{ GRUPO E } & \multirow{2}{*}{ Agua } & ECO SOCIAL ${ }^{1}$ & 56 \\
\hline & & $S A+S L^{2}$ & 1.649 \\
\hline
\end{tabular}

1. Economía Social (Cooperativas y Sociedades Laborales)

2. Sociedades Anónimas y Sociedades Limitadas

Fuente: Elaboración propia a partir de base de datos SABI y Clasificación Nacional de Actividades Económicas (CNAE - 2009).

Para este análisis se ha tenido como referencia el modelo de presentación del Estado de Valor Añadido propuesto por la Asociación Española de Contabilidad y Administración de Empresas-AECA, identificando así la distribución del valor agregado entre los diferentes factores de producción.

\section{Discusión y análisis: integración del valor social en la gestión estratégica de las organizaciones}

De acuerdo con el diseño básico del marco de referencia se trata de analizar la gestión de valor en las empresas a lo largo del ciclo estratégico, particularmente la coherencia entre las fases inicial y final del mismo: declaración de la intención estratégica y medición de resultados. Para ello, se aborda un análisis comparativo del comportamiento de dos grupos de empresas, de Economía Social y mercantiles, a lo largo del proceso de gestión estratégica. 


\subsection{El punto de partida: la intención estratégica}

En Europa, desde el lanzamiento del proyecto "La emergencia de las empresas sociales" en $1996^{2}$, que se establece como el hito de inicio de EMES (Research Network for Social Enterprise), esta Red de Investigación ha aspirado a construir un corpus teórico y empírico en torno al concepto de empresa social y otros relacionados: emprendimiento social, Economía Social, economía solidaria e innovación social ${ }^{3}$. Con este enfoque y a fin de proporcionar una construcción abstracta que permita a los grupos investigadores posicionarse en la "galaxia" de las empresas sociales (Defourny \& Nyssens, 2013), EMES identificó tres dimensiones de análisis (económica y empresarial, social y gobernanza participativa). Son dimensiones que las empresas tienden a utilizar como parte de su información corporativa y que hemos tenido en cuenta en el diseño de la estructura de análisis.

Por otro lado, en el Estudio sobre la evolución reciente de la Economía Social en Europa, el Comité Económico y Social Europeo (2018) aporta una definición recogiendo los principios básicos a los que las empresas de la Economía Social se adhieren y clarifica cuáles son los agentes de la Economía Social distinguiendo dos grandes grupos: aquellos que siguen procesos de mercado como una actividad instrumental para lograr sus fines y los que no operan en el mercado.

Esta distinción, junto con la identificación de las dimensiones de análisis, resulta relevante al diseñar el análisis comparativo entre empresas de la Economía Social y empresas mercantiles. A fin de posibilitar la comparabilidad, entre las empresas de Economía Social se analizan aquellas que siguen procesos de mercado y, específicamente, las cooperativas y las sociedades laborales, dos de las familias de la Economía Social en España. En conjunto, las cooperativas y las sociedades laborales representan el 68\% del total de entidades y el 70,5\% de los empleos directos e indirectos de la Economía Social en España. ${ }^{4}$

Las empresas seleccionadas para el análisis lo han sido de acuerdo con criterios de dimensión (empresas con mayor cifra de negocios en el año 2018 en cada grupo y en cada uno de los sectores, a partir de la información de la base de datos SABI). De esta forma se ha dispuesto de información de un mínimo de 6 empresas por cada grupo y sector siempre que ha sido posible ya que en algunos sectores las empresas de Economía Social existentes no disponen de website corporativa ni comercial. El análisis de 60 empresas, tanto de la Economía Social como mercantiles permite identificar la integración de algunos conceptos de forma recurrente en ambos tipos de organizaciones y en las tres dimensiones analizadas (actividad productiva $y$ de mercado, compromiso social y modelo de empresa y empleo).

El foco de análisis está puesto en los sectores agroalimentario y manufacturero. El alcance y la estructuración de las websites de las empresas de estos dos sectores en los grupos de Economía Social y mercantiles son semejantes, lo que ha permitido un análisis semántico-pragmá-

2. EMES Network Strategic Plan 2019-2021, chapter 2: A historical perspective on the development of EMES (disponible en www.emes.net en marzo de 2020).

3. Misión de EMES (EMES Network Strategic Plan 2019-2021).

4. Datos de CEPES (cepes.es/social/estadísticas), 2018 (consulta realizada en abril de 2020) 
tico de mayor detalle. En el resto de sectores, las diferencias no sólo de modelo de empresa y de dimensión sino incluso de modelo de negocio se trasladan a la información de la website, de alcance corporativo en el caso de las empresas mercantiles y más bien de carácter comercial en el caso de las empresas de Economía Social de la muestra, lo que conlleva diferencias de contenidos, presentación y alcance que han dificultado la comparabilidad.

En la lectura de los mapas semánticos (Figuras 2 a 7) presentados a lo largo de este capítulo los conceptos de la columna central están ubicados en función de dos criterios: i) Frecuencia de menciones totales, que es un máximo de 16 en las empresas agroalimentarias (8 de Economía Social +8 mercantiles) y de 18 en el caso de las manufactureras ( 9 de cada grupo). A más menciones, los conceptos se sitúan en una posición superior en el mapa. ii) Frecuencia de menciones en uno u otro grupo. Si hay más menciones en el grupo de empresas mercantiles, el concepto se sitúa más a la derecha. Si hay más menciones en el grupo de empresas de Economía Social, se sitúa más a la izquierda. Por otro lado, a la izquierda de la Figura se pueden leer ejemplos del léxico utilizado por las empresas de Economía Social mientras que a la derecha se pueden leer muestras del léxico utilizado por las empresas mercantiles.

\section{Empresas agroalimentarias}

En el primero de los bloques de información, relativo a la actividad productiva y de mercado (Figura 2) la tendencia a la homogeneización de mensajes en las declaraciones de ambos grupos de empresas -mercantiles o de Economía Social- se concreta en la identificación de siete campos semánticos (calidad, innovación, crecimiento y dimensión, eficiencia y rentabilidad, seguridad alimentaria, internacional y tecnología). Si bien hay diferencias en la utilización del léxico, no suponen diferenciación de conceptos. De acuerdo con estas declaraciones; en general, tanto las empresas mercantiles como las de la Economía Social son empresas de dimensión, con aspiraciones de crecimiento y una vocación por el desarrollo de estrategias orientadas a la eficiencia, la calidad, la innovación y el desarrollo de la tecnología. Así mismo, debido a los rasgos propios del sector de análisis aparece en varias empresas la mención expresa a la seguridad alimentaria. En otros casos, si bien no se hace explícita, podría considerarse inherente al concepto de calidad. 
Figura 2. Análisis semántico de las declaraciones de intención estratégica en torno a la actividad empresarial y de mercado en empresas agroalimentarias mercantiles y de Economía Social

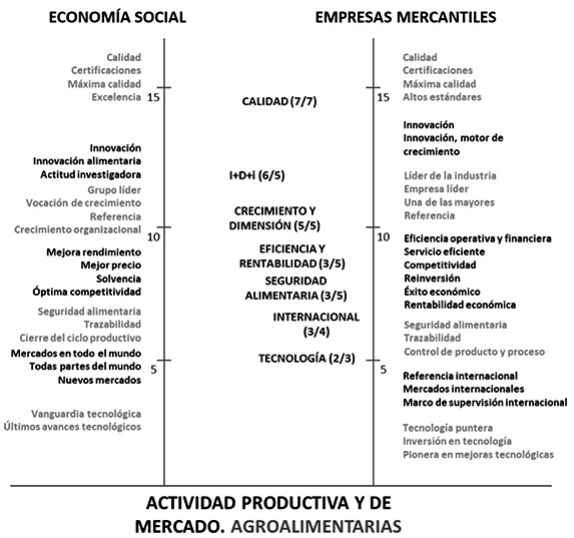

Figura 3. Análisis semántico de las declaraciones de intención estratégica en torno al compromiso social en empresas agroalimentarias mercantiles y de Economía Social

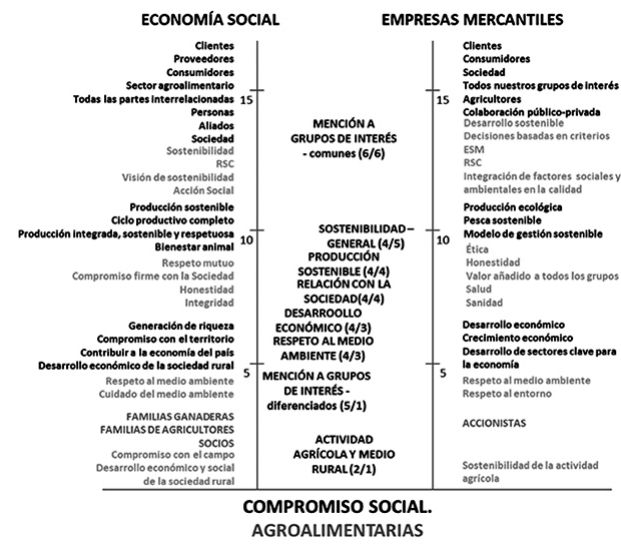

En el análisis del segundo bloque de información (compromiso social, Figura 3) surgen de nuevo siete conceptos en torno a los cuales coinciden las empresas de Economía Social y las mercantiles. Uno de ellos (Grupos de interés) puede desdoblarse en 2 -aquellos coincidentes y los que no lo son. Es aquí donde surgen unas primeras diferencias que van más allá del léxico y apuntan a un modelo diferente. Sólo en las cooperativas agroalimentarias se menciona a las familias y a los socios y sólo en las mercantiles a los accionistas.

El resto de campos semánticos contienen un léxico asimilable. Las empresas de ambos grupos manifiestan su compromiso con la sostenibilidad en general, el mantenimiento de relaciones con la Sociedad, su contribución al desarrollo económico y el respeto al medio ambiente. Además, y como conceptos específicos del sector surgen la producción sostenible y la actividad agrícola y el medio rural. Desde esta perspectiva existe, por lo tanto, una tendencia a la homogeneización de planteamientos.

La tercera perspectiva de análisis es la denominada Modelo de empresa y Empleo (Ver Figura 4). Describir la naturaleza jurídica o el modelo de empresa como un elemento de su presentación significa que se entiende como un elemento significativo en la descripción de su identidad. En ambos grupos se considera así y en el caso de la Economía Social la propia mención (o ausencia de mención) al hecho cooperativo es un aspecto relevante. Las empresas analizadas, se describen además como cooperativa de primer grado o segundo grado, trasladando en este caso la idea de la integración cooperativa. 
En relación con el empleo, sin embargo, en la muestra analizada no se identifican diferencias entre ambos grupos. Incluso son más las empresas mercantiles que manifiestan expresamente su objetivo de crear empleo, haciendo referencias a rasgos diferenciadores como estable y digno, términos próximos a los de empleo decente y sostenible, analizado en relación con las políticas de fomento de la Economía Social (Canalda, 2019) y a la forma en que se recoge en la propia Ley de Economía Social como elemento integrador de sus principios -"empleo estable y de calidad". En la misma línea las menciones relacionadas con las condiciones y entorno de trabajo y los equipos y el talento son similares. Con diferente léxico pero el mismo mensaje de fondo, en ambas se pone de manifiesto el reconocimiento a las personas y a los equipos, la cualificación o el desarrollo profesional. Habida cuenta de que las diferencias explícitas en la formulación de la intención estratégica en este grupo están referidas al propio modelo quedaría pendiente analizar de qué manera se manifiestan y si tras declaraciones semejantes existen comportamientos o resultados que puedan demostrarse como diferentes.

\section{Figura 4. Análisis semántico de las declaraciones de intención estra- tégica en torno al Modelo de empresa y Empleo en empresas agroali- mentarias mercantiles y de Economía Social}

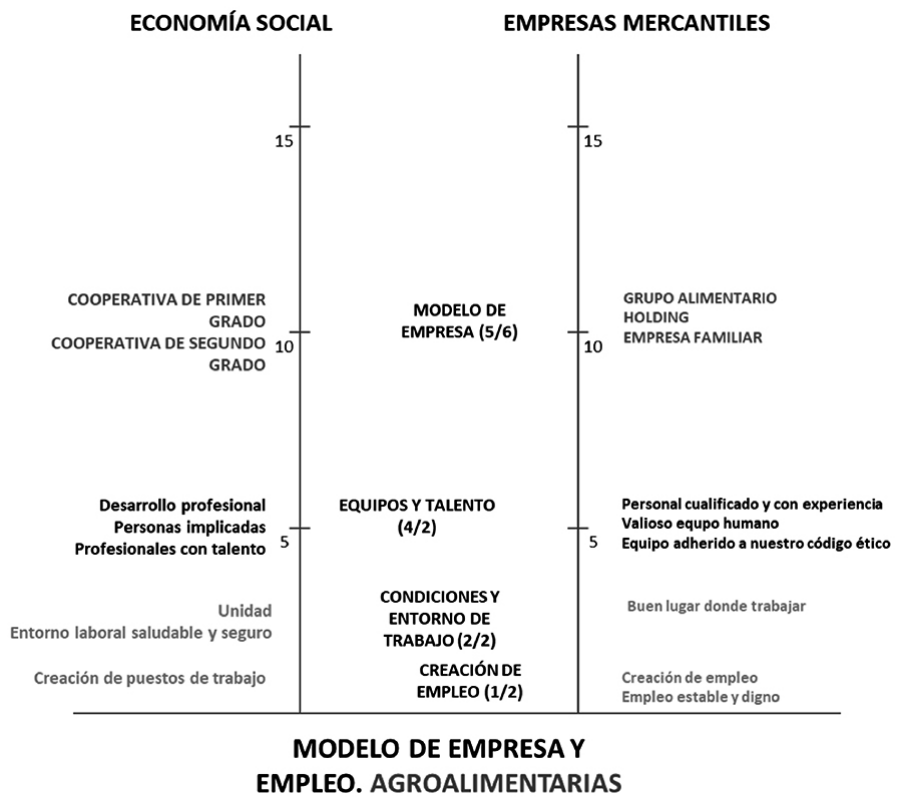




\section{Empresas manufactureras}

La Figura 5 muestra los 6 campos semánticos considerados desde la perspectiva de la actividad productiva y de mercado. Se trata de ámbitos comunes a ambos grupos, a través de los cuales se refleja la aportación de una propuesta de valor competitiva. Aspectos como la calidad, la innovación, la tecnología o la eficiencia están integrados de forma ligeramente más explícita en la comunicación de la intención estratégica de las empresas de Economía Social de la muestra. Son empresas que se presentan como líderes en un mercado global, de forma similar a las mercantiles. El aspecto en el que en la muestra analizada las empresas mercantiles ponen más énfasis frente a las de la Economía Social, y que podría relacionarse con la generación de valor, es la marca.

En la Figura 6 se identifican campos semánticos similares entre los dos grupos analizados. La mención diferenciada a algunos grupos de interés ocurre en pocos casos. Se habla de vecinos y compañeros en el caso de alguna empresa de Economía Social, lo que surge como un contrapunto de cercanía a lo local que equilibra la dimensión internacional y; por otro lado, se habla de accionistas y ONGs en el caso de las mercantiles.

Figura 5. Análisis semántico de las declaraciones de intención estratégica en torno a la actividad empresarial y de mercado en empresas manufactureras mercantiles y de Economía Social

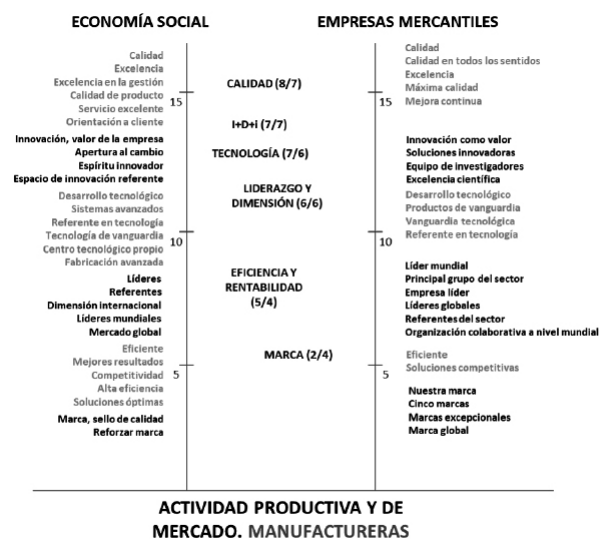

Figura 6. Análisis semántico de las declaraciones de intención estratégica en torno al compromiso social en empresas manufactureras mercantiles y de Economía Social

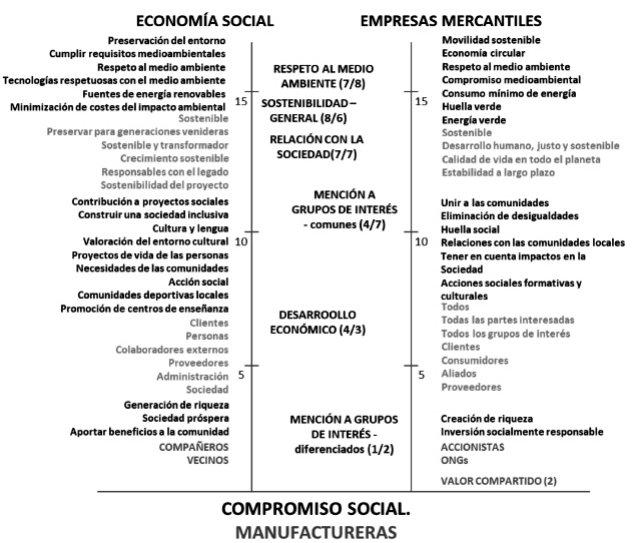

En el resto de conceptos (sostenibilidad, relación con la sociedad, desarrollo económico), las declaraciones tienen rasgos homogéneos y son agrupables en el mismo campo semántico. 


\section{Figura 7. Análisis semántico de las declaraciones de intención estraté- gica en torno al Modelo de empresa y Empleo en empresas manufac- tureras mercantiles y de Economía Social}

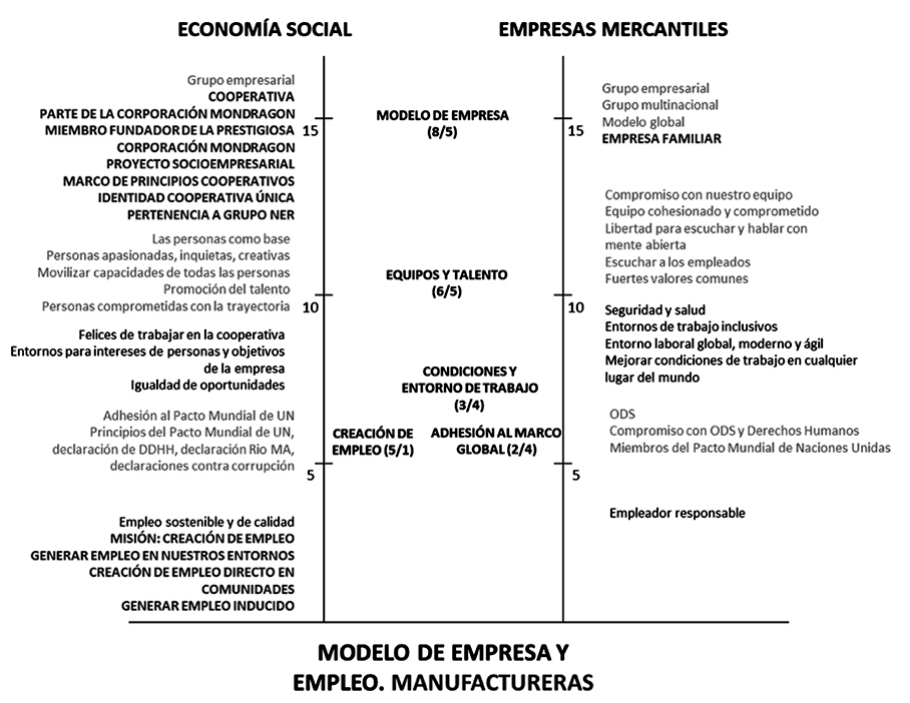

Finalmente, en la Figura 7 se identifican los campos relativos a la comparación del modelo de empresa y empleo. Por un lado, las empresas de Economía Social tienden a señalar como parte del modelo de gobernanza su realidad cooperativa, su pertenencia a grupos cooperativos o la adhesión al marco de principios cooperativos. Entre las empresas mercantiles se destaca, además de temas comunes, la naturaleza de empresa familiar, cuando lo es.

Así mismo, aparece de forma diferenciada la mención explícita a la creación de empleo como objetivo de las empresas de Economía Social. Hay que tener en cuenta que en el caso de todas las manufactureras se trata de cooperativas de trabajo asociado, lo que explica las diferencias con respecto a las cooperativas agroalimentarias, en las que la creación de empleo no se explicita de la misma manera en las websites analizadas.

Las declaraciones en torno a los campos semánticos de equipos y talento y condiciones y entorno de trabajo vuelven a tener un peso similar en ambos grupos y surge, además, la adhesión al marco global como un elemento adicional que integran en su modelo tanto las empresas de Economía Social como las mercantiles, especialmente estas últimas en la muestra analizada.

Todo lo anterior abre la cuestión sobre si en el primer elemento de este marco de referencia, las diferencias en las declaraciones de la intención estratégica son un rasgo distintivo fundamental entre empresas de la Economía Social y las empresas mercantiles. En el contexto actual, la declaración de una finalidad social per se no evidencia una gestión diferenciada del valor social por parte de las empresas de Economía Social con respecto a las mercantiles. Ade- 
más, al haberse convertido en elementos importantes para su reputación, las empresas más grandes tienden a tener recursos especializados para la comunicación. En este sentido, Social Economy Europe plantea en su Libro Blanco de la Economía Social las limitaciones de la RSC en la medida en que no es una herramienta obligatoria ni vinculada a la misión de la empresa y está "mal utilizada por parte de algunas corporaciones con una finalidad de marketing”.

\subsection{Cerrando el ciclo: De la intención estratégica a la medición de los resultados}

Detrás de todo lo anterior surge la cuestión que Gast et al. (2020) denominan el purpose gap, que refleja la distancia entre la declaración de un propósito y el efecto real que llega a tener. Tal como se ha visto en el análisis comparativo de la intención estratégica de las empresas de ambos grupos, el concepto más claramente diferenciador de la Economía Social hace referencia al modelo de empresa y a la adhesión a los principios de la Economía Social. Estos principios están establecidos en España en la Ley 5/2011 de Economía Social: "a) Primacía de las personas y del fin social sobre el capital, (...) que lleva a priorizar la toma de decisiones más en función de las personas y sus aportaciones de trabajo (...) o en función del fin social, que en relación a sus aportaciones al capital social; b) Aplicación de los resultados obtenidos de la actividad económica principalmente en función del trabajo aportado y servicio o actividad realizada por las socias y socios o sus miembros y, (...) al fin social objeto de la entidad; c) Promoción de la solidaridad interna y con la sociedad que favorezca el compromiso con el desarrollo local, la igualdad de oportunidades entre hombres y mujeres, la cohesión social, la inserción de personas en riesgo de exclusión social, la generación de empleo estable y de calidad, la conciliación de la vida personal familiar y laboral y la sostenibilidad; y d) Independencia de los poderes públicos".

Estos principios están integrados en el modelo de empresa de Economía Social. "La propia naturaleza y principios de actuación de las empresas de Economía Social en general, y particularmente de las cooperativas, las erigen como exponentes representativos de una empresa responsable, modelo y vanguardia de la nueva manera de hacer economía, donde sus acciones atienden al resultado económico y financiero, pero también al social y al ambiental." (Sajardo-Moreno, 2017).

En línea con estas reflexiones, cabe cuestionar si más allá de la adhesión a los principios pudiera evidenciarse alguna diferencia de las empresas de Economía Social con respecto a empresas mercantiles en los resultados obtenidos. Toda vez que no existen sistemas de información social que se utilicen de forma universal, se ha abordado un análisis de la distribución del valor añadido generado en empresas mercantiles y empresas de Economía Social en los diferentes sectores de actividad (ver tabla 2. Ficha de análisis). Esta distribución se concreta en las retribuciones que la empresa destina a los distintos factores de producción, ya sea en forma de salarios, impuestos, intereses o resultado (repartido o no).

5. Libro Blanco de la Economía Social (2015). "La Economía Social retoma la iniciativa" 
Azcárate et al. (2013), señalan que "la distribución del valor añadido resulta como un juego de suma cero, es decir, en el momento en que uno de los agentes aumenta su porción de valor añadido recibido supone la reducción de la porción recibida por algún otro". De esta manera, y de acuerdo con los principios de la Economía Social, cabría suponer diferencias entre las retribuciones del factor trabajo en las empresas de Economía Social y aquellas de carácter mercantil, consecuencia de una estrategia orientada por estos principios.

El análisis comparado entre empresas de Economía Social y empresas mercantiles en los cinco grupos CNAE (ver tabla 6) apunta a una mayor retribución al factor trabajo en las empresas de Economía Social, si bien el comportamiento en cada sector es diferente.

\section{Tabla 6. Comparativa de la distribución del valor añadido entre los dife- rentes factores de producción, valor agregado y empleo medio* en em- presas de Economía Social y empresas mercantiles de 5 grupos CNAE, ejercicio 2018}

\begin{tabular}{|c|c|c|c|c|c|c|c|c|c|c|c|}
\hline \multirow[b]{2}{*}{$\begin{array}{l}\text { Grupo } \\
\text { CNAE }\end{array}$} & \multirow[b]{2}{*}{ Descripción } & \multirow[b]{2}{*}{ Tipología } & \multirow[b]{2}{*}{$\begin{array}{c}\text { Valor } \\
\text { Agregado } \\
\text { (Miles } € \text { ) }\end{array}$} & \multirow[b]{2}{*}{$\begin{array}{l}\text { Comparativa } \\
\text { VA generado } \\
\text { (ES/merc) }\end{array}$} & \multicolumn{5}{|c|}{ DISTRIBUCIÓN DEL VALOR AÑADIDO } & \multirow[b]{2}{*}{$\begin{array}{l}\text { EMPLEOO } \\
\text { MEDIO }\end{array}$} & \multirow[b]{2}{*}{$\begin{array}{c}\text { Comparativa } \\
\text { Empleo } \\
\text { medio } \\
\text { (ES/merc) }\end{array}$} \\
\hline & & & & & $\begin{array}{c}\text { GASTOS } \\
\text { PERSONAL }\end{array}$ & $\begin{array}{c}\text { GASTOS } \\
\text { FINANCIIROS } \\
\text { YASIMILADOS }\end{array}$ & $\begin{array}{l}\text { IMPUESTOS } \\
\text { SOBRE } \\
\text { SOCIEDADES }\end{array}$ & $\begin{array}{c}\text { AMORTIZA- } \\
\text { CIONES } \\
\text { INMOVILI-ZADO }\end{array}$ & RESULTADOS & & \\
\hline \multirow[t]{2}{*}{ GRUPOA } & \multirow[t]{2}{*}{ Agro } & $\begin{array}{l}\text { ECO } \\
\text { SOCIAL } \\
\text { SA+SL }\end{array}$ & 478 & \multirow[t]{2}{*}{$158 \%$} & \multirow{2}{*}{$\begin{array}{l}57 \% \\
56 \% \text {. }\end{array}$} & \multirow[t]{2}{*}{$\begin{array}{r}3 \% \\
3 \% \\
\end{array}$} & \multirow{2}{*}{$\begin{array}{l}5 \% \\
.5 \%\end{array}$} & \multirow{2}{*}{$\begin{array}{l}17 \% \\
16 \%\end{array}$} & \multirow{2}{*}{$\begin{array}{l}18 \% \\
19 \%\end{array}$} & 13 & \multirow[t]{2}{*}{$130 \%$} \\
\hline & & $S A+S L$ & 303 & & & & & & & 10 & \\
\hline \multirow[t]{2}{*}{ GRUPO B } & \multirow[t]{2}{*}{ Extractivas } & $\begin{array}{l}\text { ECO } \\
\text { SOCIAL }\end{array}$ & 1.308 & \multirow[t]{2}{*}{$60 \%$} & \multirow{2}{*}{$\begin{array}{l}67 \% \\
25 \%\end{array}$} & \multirow[t]{2}{*}{$1 \%$} & \multirow{2}{*}{$\begin{array}{c}5 \% \\
36 \%\end{array}$} & \multirow{2}{*}{$\begin{array}{l}17 \% \\
.16 \% .\end{array}$} & \multirow{2}{*}{$\begin{array}{l}10 \% \\
15 \% \\
\end{array}$} & 5 & \multirow[t]{2}{*}{$33 \%$} \\
\hline & & $S A+S L$ & 2.176 & & & & & & & 15 & \\
\hline \multirow[t]{2}{*}{ GRUPO C } & \multirow{2}{*}{$\begin{array}{l}\text { Manufacturer } \\
\text { as }\end{array}$} & $\begin{array}{l}\text { ECO } \\
\text { SOCIAL } \\
\end{array}$ & 1.383 & \multirow[t]{2}{*}{$145 \%$} & \multirow{2}{*}{$\begin{array}{l}70 \% \\
62 \% .\end{array}$} & \multirow[t]{2}{*}{$2 \%$} & \multirow{2}{*}{$\begin{array}{l}1 \% \\
6 \% \\
\end{array}$} & \multirow{2}{*}{$\begin{array}{l}13 \% \\
.13 \% .\end{array}$} & \multirow{2}{*}{$\begin{array}{l}14 \% \\
.17 \% .\end{array}$} & 25 & \multirow[t]{2}{*}{$147 \%$} \\
\hline & & $S A+S L$ & 956 & & & & & & & 17 & \\
\hline \multirow[t]{2}{*}{ GRUPO D } & \multirow[t]{2}{*}{ Energía } & $\begin{array}{l}\text { ECO } \\
\text { SOCIAL }\end{array}$ & 446 & $40 \%$ & $48 \%$ & $2 \%$ & $6 \%$ & $22 \%$ & $22 \%$ & 8 & $100 \%$ \\
\hline & & $S A+S L$ & 1.125 & & $26 \%$ & $.12 \%$ & 50 & $24 \%$ & $34 \%$ & 8 & \\
\hline GRUPOE & 'Agua & $\begin{array}{l}\text { ECO } \\
\text { SOCIAL }\end{array}$ & 147 & $4 \%$ & $74 \%$ & $1 \%$ & $3 \%$ & $16 \%$ & $5 \%$ & 6 & $10 \%$ \\
\hline 1. & & $S A+S L$ & 3.659 & & $53 \%$ & $.5 \%$ & $2 \%$ & $15 \%$ & $.25 \%$ & 59 & \\
\hline
\end{tabular}

* Sobre fondo gris, valores en los que el grupo de empresas de Economía Social obtiene una media superior

Fuente: Elaboración propia a partir de información de la base de datos SABI

Centrándonos nuevamente en las empresas agroalimentarias y las manufactureras, cabe destacar que en el Grupo A (Agricultura, ganadería, silvicultura y pesca), la estructura de reparto del valor añadido entre los distintos factores es muy similar entre las empresas de Economía Social y las empresas mercantiles. La retribución al factor trabajo muestra una diferencia poco significativa (+1\% en favor de las empresas de Economía Social). Como datos adicionales del perfil financiero, tanto el empleo medio como el valor agregado medio son superiores en el caso de la Economía Social.

Por otro lado, en el Grupo C (Industrias manufactureras), la retribución al factor trabajo muestra una diferencia significativa entre los dos grupos de empresas comparados (70\% del valor agregado en empresas de Economía Social frente al 62\% en empresas mercantiles). Esta 
diferencia se manifiesta así mismo en el porcentaje de valor agregado distribuido a impuestos y resultados, que es significativamente superior en el caso de las empresas mercantiles. En este caso, además, tanto el valor agregado como el empleo medio son superiores en las empresas de Economía Social.

Por tanto, la estructura de distribución del valor añadido entre las empresas de Economía Social y las empresas mercantiles en el ejercicio 2018 es diferente en todos los sectores analizados en cuanto a la retribución del factor trabajo, lo que se relaciona con uno de los principios orientadores de la Economía Social (aplicación de los resultados de la actividad en función del trabajo aportado). Sin embargo, resultaría difícil informar sobre otros principios o sobre elementos en los que se sustenta el compromiso social. No se dispone de información comparable sobre el valor social generado, lo que dificulta una gestión estratégica eficaz.

Frente a los beneficios de modelos de definición y seguimiento de objetivos financieros, de mercado y gestión de los procesos internos, el menor trabajo desarrollado para la medición y gestión de indicadores de valor social limita su incorporación a los procesos de gestión estratégica. En Strategy Maps (Norton \& Kaplan, 2000), los autores presentan una adaptación del cuadro de mando integral a entidades sin ánimo de lucro, entendiendo que la primera de las perspectivas puede tener otro alcance y se redefine como la perspectiva de Resultados 0 misión. A partir de ahí, el cuadro de mando integral se ha aplicado en diferentes tipos de organizaciones. Retolaza et al. (2012) plantean un decálogo para la aplicación del cuadro de mando integral en el tercer sector. Ganga et al. (2015) lo presentan como una herramienta estratégica para el equipo directivo de una organización no gubernamental. Murillo (2020) diseña una herramienta basada en el Cuadro de Mando Integral para gestionar el impacto social en empresas de empleo inclusivo. A día de hoy, tanto la comparabilidad entre indicadores en este tipo de organizaciones como la propia aplicación sistematizada de indicadores de valor social siguen siendo muy limitadas. La disponibilidad de información comparable y estandarizada sobre el valor social generado emerge como uno de los elementos a integrar en el marco de referencia para la gestión estratégica del valor social.

Diferentes estudios han tratado de vincular la información de los estados financieros con la generación de valor social (Lazcano \& San-Jose, 2019; Iturrioz et al., 2019). En el primero de ellos se concluye que es necesaria una contabilidad social que refleje el valor generado por empresas de Economía Social y que no se refleja en la contabilidad financiera. Por otro lado, el modelo poliédrico de contabilidad social ha mostrado una aplicabilidad mayor en empresas de diversos sectores de la Economía Social (Lazcano \& Beraza, 2019). Y en la aplicación de dicho modelo, Etxezarreta et al. (2018) concluyen que es especialmente adecuado para entidades de Economía Social, debido a que las variables de valor son específicas en cada caso, lo que es coherente con la diversidad de las entidades de Economía Social.

Siendo un modelo que proporciona información monetizada, el proceso parte del diálogo con los stakeholders, lo que además de asegurar una perspectiva multistakeholder, evita el riesgo de desligar los resultados de la percepción real de valor que estos tienen, subdividiendo "un concepto complejo e intangible, como el de valor social, en una serie de factores constituyentes, denominados Variables de Valor" (Retolaza \& San-Jose, 2016). Además, toda vez que el 
proceso de contabilidad social se incorpora de forma sistemática a la gestión, proporciona la visión temporal que permite establecer conexiones entre el pasado, el presente y el futuro y, por lo tanto, generar pensamiento estratégico. Por otro lado, el modelo poliédrico contempla la generación de valor a diferentes stakeholders y desde diferentes ecosistemas (mercado o no mercado y su integración en un concepto de valor social integral), aportando la perspectiva sistémica que requiere la gestión estratégica. Así, la integración de la contabilidad social ayudaría a cerrar el ciclo y convertirse en una referencia complementaria a la contabilidad financiera para garantizar el alineamiento entre el propósito y los resultados.

\section{Conclusiones}

A lo largo de este artículo se ha presentado un marco de referencia para la integración de la contabilidad social en la gestión estratégica de las empresas de Economía Social. Este marco está estructurado en torno a las tres fases del proceso de gestión estratégica (formulación de la intención estratégica, implantación y medición de resultados) y con respecto a la primera y a la última se ha abordado un análisis comparativo entre las empresas de Economía Social y las empresas mercantiles, a través del cual se ha puesto de manifiesto la necesidad de incorporar en la gestión estratégica de las empresas de Economía Social información específica sobre el valor social generado.

Partiendo de la definición de la intención estratégica, se ha identificado en los dos grupos de empresas analizados (Economía Social y mercantil) una convergencia de conceptos en planteamientos cada vez más híbridos tanto en torno a la actividad empresarial como al compromiso social, principalmente en los sectores de análisis en los que la estrategia de negocio está dirigida a un mismo mercado objetivo, como es el caso de la actividad agroindustrial y la manufacturera, y la dimensión de las empresas es comparable. Los principales elementos de diferenciación tienen que ver con el modelo de empresa y, de hecho, una parte significativa de las empresas de la muestra dan valor en su comunicación corporativa a la realidad cooperativa y a los principios de la Economía Social. Para empresas de pequeña dimensión o pertenecientes al resto de sectores analizados (Actividad extractiva, energía y gestión de agua y residuos), el primer elemento a integrar en este marco de referencia es la propia necesidad de definir y comunicar su intención estratégica, a través de declaraciones como la misión o el propósito. Si la intención estratégica ni se explicita ni se comunica no puede gestionarse.

En relación con la medición de resultados, se ha analizado si a través de la información de la contabilidad financiera puede reflejarse alguno de los principios orientadores de la Economía Social, que en la formulación de su intención estratégica, aparecen como elementos constitutivos de un modelo de empresa diferente. Para ello, se ha abordado un análisis del estado de distribución del valor agregado en empresas mercantiles y empresas de Economía Social, concluyendo que en los cinco sectores analizados la información financiera refleja el principio orientador de primacía del trabajo sobre el capital ya que en el reparto porcentual del valor agregado la retribución al trabajo es mayor que en las empresas mercantiles. Sin embargo, 
existen otros elementos insertos en los principios inspiradores de la Economía Social, tanto sobre la relación con sus propias personas socias como, sobre todo, con el entorno, que no son visibles a través de la contabilidad financiera.

El análisis permite concluir que existe un gap importante entre la aplicación de sistemas de información orientados a la gestión de las variables puramente empresariales y las variables sociales. Esto dificulta a las empresas de la Economía Social la gestión y difusión del valor social que generan y puede poner en duda el alineamiento entre su propósito y los resultados obtenidos. La formulación de una intención estratégica con un componente social no las diferencia de las empresas mercantiles y tampoco garantiza su cumplimiento. A través de la información financiera, no se consigue identificar si se cubre este gap, lo que redunda en las dificultades para conseguir una gestión estratégica coherente. Sin una información completa, objetiva y estandarizada sobre la generación de valor social, difícilmente se puede diferenciar el valor generado por las empresas de la Economía Social o por las empresas mercantiles, ya que estas últimas han incorporado de forma generalizada información cualitativa de carácter social, que en algunos casos comunican de forma más profesional.

Desde la perspectiva empresarial, se han desarrollado diferentes variables de análisis y modelos y se ha generado una dinámica de trabajo y un lenguaje común que facilita la comprensión y la valoración de la estrategia empresarial tanto internamente como por parte de agentes externos. Sin embargo, no existe un desarrollo paralelo comparable que integre el valor social generado a través del mercado y a través de actividades de no mercado y que contemple el conjunto de los stakeholders para los que las organizaciones generan ese valor. De esta forma, el fin social declarado por las organizaciones no encuentra un reflejo claro en su gestión estratégica, No es fácil para los agentes externos visibilizar la estrategia social de las organizaciones ni es fácil para las propias organizaciones transmitir el alcance de su estrategia de forma transparente y fiable.

En esta situación diversas investigaciones han señalado la adecuación del modelo poliédrico de la contabilidad social para reflejar la realidad de la actividad de la Economía Social que no es visible a través de la información financiera. Teniendo en cuenta la lógica del proceso de gestión estratégica, este artículo ha incorporado una visión novedosa al considerar su integración en diferentes fases de tal proceso. En relación con la fase inicial, el modelo poliédrico parte de la identificación de las variables de valor, incorporando así un elemento descriptivo a partir de la percepción de valor de los stakeholders. En la fase de seguimiento de resultados, permite medir los de valor social, monetizándolos y haciéndolos comprensibles y comparables. Por ello, teniendo en cuenta la perspectiva de análisis de este artículo, se propone la incorporación de la contabilidad social y el modelo poliédrico en el marco de referencia para la integración del valor social en la gestión estratégica y el análisis de la coherencia entre la intención estratégica y los resultados.

La investigación presenta algunas limitaciones. En primer lugar, el análisis de la estructura de generación y distribución de valor añadido se ha limitado a un solo ejercicio, faltando por lo tanto un análisis de la dinámica evolutiva de los indicadores de referencia. Por otro lado, el nivel de agregación de las empresas de la muestra es amplio y un mismo grupo CNAE podría 
incluir realidades diferentes. Así mismo, el análisis de las empresas de Economía Social ha incluido las cooperativas y las sociedades laborales como entidades más representativas de la Economía Social en número y dimensión; quedando fuera, por lo tanto, otras iniciativas empresariales como los Centros Especiales de Empleo y Empresas de Inserción, en la medida en que no adopten alguna de las formas jurídicas mencionadas. Así mismo, a futuro podría plantearse el análisis en otros sectores de actividad en los que la Economía Social está presente.

En un marco global en el que la Agenda 2030 de las Naciones Unidas en los entornos institucionales y empresariales y la correlación entre la actividad empresarial y la sostenibilidad se han hecho más patentes, las empresas de Economía Social necesitan avanzar en la incorporación del valor social a su gestión estratégica. A futuro se abre una vía de investigación para el desarrollo de un modelo que, a partir del marco de referencia aquí propuesto, incorpore las cuestiones, procesos y herramientas clave para la efectiva integración del valor social en la gestión estratégica de las organizaciones que manifiestan una aspiración a generar un valor social, de tal manera que puedan medirlo, comunicarlo y gestionarlo estratégicamente. De esta forma, así mismo, las instituciones y la sociedad podrán evaluar la aportación de valor social que a las organizaciones se les demanda.

\section{Bibliografía}

AUSTIN, J., STEVENSON, H. \& WEI-SKILLERN, J. (2006): "Social and commercial entrepreneurship: same, different, or both?", Entrepreneurship theory and practice, 30(1), 1-22.

AZCÁRATE LLANES, F. \& FERNÁNCEZ CHULIÁN, M. (2013): “El Estado del Valor Añadido: una propuesta alternativa para la diferenciación entre los posicionamientos Bruto y Neto". Revista Contable, 16, 40-51.

BOWER, J. \& GILBERT, C. (2005): From resource allocation to Strategy, Oxford University press.

CANALDA CRIADO, S. (2019) "El fomento del empleo decente y sostenible en cooperativas y sociedades laborales". REVESCO, Revista de Estudios Cooperativos, 132, 77-96.

CHENHALL, R.H. (2005): "Integrative strategic performance measurement systems, strategic alignment of manufacturing, learning and strategic outcomes: an exploratory study", Accounting, organizations and society, 30(5), 395-422

CHIN, M.K., HAMRICH, D.C. \& TREVIÑO, L.K. (2013): "Political ideologies of CEOs: The influence of executives' values on corporate social responsibility", Administrative Science Quarterly, 58:197-232.

COLLIS, D.J. \& MONTGOMERY, C.A. (1995): "Competing on Resources: Strategy in the 1990s". Knowledge and strategy, 25-40. 
DE CASTRO SANZ, M. (2006): "Las empresas de economía social y la responsabilidad social corporativa", Papeles de Economía Española, 108, 92-104.

DEFOURNY, J., NYSSENS, M. (2013): "El enfoque EMES de empresa social desde una perspectiva comparada”, EMES Working paper Series, 13/01.

DRUCKER, P.F. (1986): Management. Tasks, responsibilities, practices, Truman Tally Books. New York, pp. 14-24.

ETXEBARRIA, I.A. (2009): "Las memorias de sostenibilidad: un instrumento para la gestión de la sostenibilidad", Revista española de financiación y contabilidad, 677-697.

ETXEZARRETA, E., MENDIGUREN, J.C.P., DIAZ, L. \& ERRASTI, A. (2018): "Valor social de las cooperativas sociales: aplicación del modelo poliédrico en la cooperativa para la acogida de menores Zabalduz, S.Coop.", CIRIEC-España, Revista de Economía Pública, Social y Cooperativa, (93), 155-180, DOI: 1.7203/CIRIEC-E.93.9953.

FRANCÉS, A. (2006): Estrategia y planes para la empresa: con el cuadro de mando integral, Pearson Educación.

FRIEDMAN, M. (1970): The Social Responsibility of Business Is to Increase Its Profits, The New York Times Magazine.

GANGA, F., RAMOS, E., LEAL, A. \& PÉREZ, K. (2015): "Strategic Administration: Application of the Balanced Scorecard (BSC) To a Non-Governmental Organization", Revista de Ciencias Sociales, XXI, 1, 136-159.

GAST, A., ILLANES, P., PROBST, N., SCHANINGER, B. \& SIMPSON, B. (2020): “Purpose: Shifting from why to how". McKinsey Quarterly, April 2020.

GUPTA, A., BRISCOE, F. \& HAMBRICK, D.C. (2018): “Evenhandedness in resource allocation: Its relationship with CEO ideology, organizational discretion and firm performance", Academy of Management Journal, 61(5), 1848-1868.

HAMEL, G. \& PRAHALAD, C.K. (1990): "El propósito estratégico", Harvard Business School Review, 1, 75-90.

HITT, MICHAEL A., et al. (1995): "Understanding strategic intent in the global marketplace", Academy of Management Perspectives, 9 (2), 12-19.

HOPE, J. \& FRASER, R. (2003): Beyond budgeting: How managers can break free from the annual performance trap, Harvard Business Press. 
ITURRIOZ DEL CAMPO, J., MASA LORENZO, C.I. \& MEDINA CASTAÑO, A. (2019): "La transparencia sobre la Responsabilidad Social Corporativa y su implicación con el comportamiento económico-financiero: efectos en las empresas de Economía Social", REVESCO, Revista de Estudios Cooperativos, tercer cuatrimestre, 132, 122-143.

JOHNSON, G., SCHOLES, K. \& WHITTINGTON, R. (2006): Dirección estratégica, 7ạ ed. Prentice Hall.

LAZCANO, L., SAN-JOSE,L. (2019): "Monetización del valor social: el caso CLADE”, Gizarte Ekonomiaren Euskal Aldazkaria/Revista Vasca de Economía Social, 16, 103-127. DOI: 10.1387/revés.20903.

LAZCANO, L., SAN-JOSE, L. \& RETOLAZA, J. (2019): "Social Accounting in the Social Economy: A Case Study of Monetizing Social Value", Modernization and Accountability in the Social Economy Sector, May 2019, 132-150.

LIEDTKA, J.M. (1998): "Linking strategic thinking with strategic planning", Strategy \& Leadership, 26(4), 30.

MARIE LAUESEN, L. (2013): "CSR in the aftermath of the financial crisis", Social Responsibility Journal, 9(4), 641-663.

MARITAN, C.A. \& LEE, G.K. (2017): "Resource allocation and Strategy", Journal of Management, 43(8), 2411-2420. DOI.0RG/10.1177/0149206317729738.

MARSDEN, G., KELLY, C. \& SNELL, C. (2006): "Selecting indicators for strategic performance management", Transportation research record, 1956(1), 21-29.

MINTZBERG, H. (1990): "Strategy formation: Schools of thought", Perspectives on strategic management", 105-235.

MONZÓN, J. \& CHAVES, R. (2016): Recent evolutions of the Social Economy in the European Union. European Economic and Social Committee, CIRIEC International.

MURILLO, L.M. (2020): “Cuadro de mando integral para la gestión del impacto social en organizaciones de empleo inclusivo", CIRIEC-España, Revista de Economía Pública, Social y Cooperativa, 98, 153-18. DOI: 107203/CIRIEC-E.98.13368.

NODA, T. \& BOWER, J. (1996). "Strategy as iterated processes of resource allocation", Strategic Management Journal, 17, Special Issue: Evolutionary Perspectives on Strategy, 159-92.

NORTON, D. \& KAPLAN, R. (2000): Strategy Maps. Converting intangible assets into tangible outcomes. Harvard Business. 
ODITA, A. \& BELLO, A. (2015): "Strategic intent and organizational performance: A study of banks in Asaba, Delta State Nigeria", Information and Knowledge Management, 60-71

PEARCE, J.A \& ROBINSON, R.B (2011): Strategic Management: Formulation, Implementation and control, 12th ed., MC Graw Hill.International.

PEDREÑO, J.A. (2017): "Reflexiones, sinergias y clarificación sobre nuevos conceptos y modelos: Economía social, empresa social, emprendimiento social, economía del bien común, economía solidaria y economía colaborativa", Revista española del tercer sector, 35, 45-72.

PETERAF, M.A. (1993): "The cornerstones of competitive advantage: a resource-based view", Strategic Management Journal, 14(3), 1, 179-191.

PINTADO, T. \& SÁNCHEZ-HERRERA, J. (2012): Nuevas tendencias en comunicación, ESIC Editorial.

PORTER, M.E. \& KRAMER, M.R. (2006): "Strategy and society: the link between corporate social responsibility and competitive advantage", Harvard business review, 84(12), 78-92.

RETOLAZA, J.L., PRUÑONOSA, J. T. \& SAN-JOSE, L. (2012): "El cuadro de mando integral en las Entidades No Lucrativas", Revista de Contabilidad y Dirección, 14, 89-106.

RETOLAZA, J.L. \& SAN-JOSE, L. (2016): “Contabilidad Social para la Sostenibilidad. Modelo y aplicación", Revista de Contabilidad y Dirección. 23, 159-178.

RETOLAZA, J. L. \& SAN-JOSE, L. (2018): “Contabilidad Social para el Bien Común”, Revista de responsabilidad social de la empresa, 29, 95-122.

SAJARDO-MORENO, A. \& CHAVES-SAJARDO, R.J. (2017): "Social responsibility in social economy companies: A comparative analysis of the productive fabric of the Valencian Community", REVESCO, Revista de Estudios Cooperativos, 125, 213-242.

SOCIAL ECONOMY EUROPE (2015): Libro blanco de la Economía Social. La Economía Social retoma la iniciativa.

SOLÓRZANO, M., GUZMÁN, C., SAVALL, T. \& VILLAJOS, E. (2018): "Identidad de la empresa social en España: análisis desde cuatro realidades socioeconómicas", CIRIEC-España, Revista de Economía Pública, Social y Cooperativa, 92, 155-182. DOI:org/107203/CIRIEC-E92.9236. 